\title{
Development of a Teaching Aid for Teaching Dynamic Motion Using the Tango Platform
}

\author{
Yuki Aoki* and Izuho Funatsu
}

(Received 12 June 2018 and accepted in revised form 8 December 2018)

\begin{abstract}
We developed a teaching aid for teaching dynamic motion using a marker-less AR device based on the Tango platform. The mass point motion along a real potential is rendered in an augmented reality space. This teaching aid enables learners to observe a mass point motion in any potential. The accuracy of the motion measurements was evaluated based on the results of uniformly accelerated motion along the slopes and free-fall motion.
\end{abstract}

Keywords: dynamics, velocity, augmented reality, Tango, smartphone

\section{Introduction}

With the recent improvements in processing speeds of smartphone image recognitions, the use of markerbased augmented reality (AR) for educational purposes has increased significantly ${ }^{(1-5)}$. Using the AR marker embedded in textbooks, a three-dimensional AR object can be treated as a supplemental teaching aid ${ }^{(6)}$. However, a major drawback associated with markerbased AR is that it does not correspond to the real space because the AR environment is rendered using markers for references. This reduces the efficacy of the applications. On the other hand, a marker-less AR application, which does not require the marker, has high versatility. Realizing a marker-less AR application that corresponds with real space requires accurate spatial perception. In recent years, HoloLens ${ }^{(7)}$ developed by Microsoft, has attracted significant attention as a device that can be utilized in marker-less AR applications ${ }^{(8)}$. Unfortunately, HoloLens is still expensive and hence is not economically viable for individual learners and practical educational applications. The Tango platform whose technology is currently taken over as ARCore ${ }^{(9)}$, has shown promise for marker-less AR applications. Tango is a smartphone-based platform; therefore, implementing this platform in Information and Communication Technology (ICT) education applications is relatively simple.

In this study, we developed a teaching aid that can be used in dynamic motion experiments. The proposed system employs the Tango platform for realizing a markerless AR application. The use of a dynamics cart is a wellknown experimental method for conventional dynamic motion experiments. The dynamics cart is moved along a

\footnotetext{
* Faculty of Education, Gunma University, Japan
}

slope, and then a distance marked on a tape is measured at fixed time intervals, thereby enabling the determination of the velocity changes of an object on the slope. However, in this conventional experimental method, the teaching aid must be customized for each experiment. The usable potential forms are limited to unidirectional motion measurements. Moreover, it is difficult to track the rapid motion of the dynamics cart in real time. Thus, by using a Tango device to recognize the potential form in real space and displaying the motion of a sphere displayed in AR (AR sphere) within the recorded potential, it may be possible to realize object motion changes in a manner that is easily understood by learners.

To that effect, we developed a teaching aid, wherein an AR sphere moves within the real space potential. We also evaluated the accuracy of measurements for both uniformly accelerated motion along the slopes and free-fall motion.

\section{Development of Proposed Teaching Aid}

\subsection{Tango Devices}

The teaching aid was developed using a Zenfone AR (ZS571KL) smartphone developed by ASUS, which is based on the Tango platform ${ }^{(10)}$. Zenfone is equipped with a TOF-type depth camera; thus, by taking measurements in combination with an inertial measurement unit (IMU), a spatial perception of real space can be realized.

\subsection{Development Environment}

The software was developed on the Unity 5.6 by 
importing "Tango SDK." By replacing default "Camera" to "Tango Camera," the software can be developed like the conventional marker-based AR software.

\subsection{Software Specifications}

When the software is executed, the potential in real space photographed by a rear-facing camera is recognized (inset in Figure 1(a)). Tapping the screen displays an AR sphere, and the motion of the AR sphere within the real space potential is rendered (Figure 1(b)).

The spatial perception of potential is estimated using a depth camera installed on the rear-side of the device, which measures distance from each position, and then creates a point cloud in the real space potential. The time required for gathering the point cloud data shown in Figure 1(a) is approximately $5 \mathrm{~s}$. The AR sphere is rotated according to the physics engine in UNITY 5.6. The physics engine is configured such that the AR sphere is regarded as a point mass, and the momentum is ignored. The friction between the potential surface and the AR sphere, and the coefficient of restitution with the object was set to zero. The gravita-
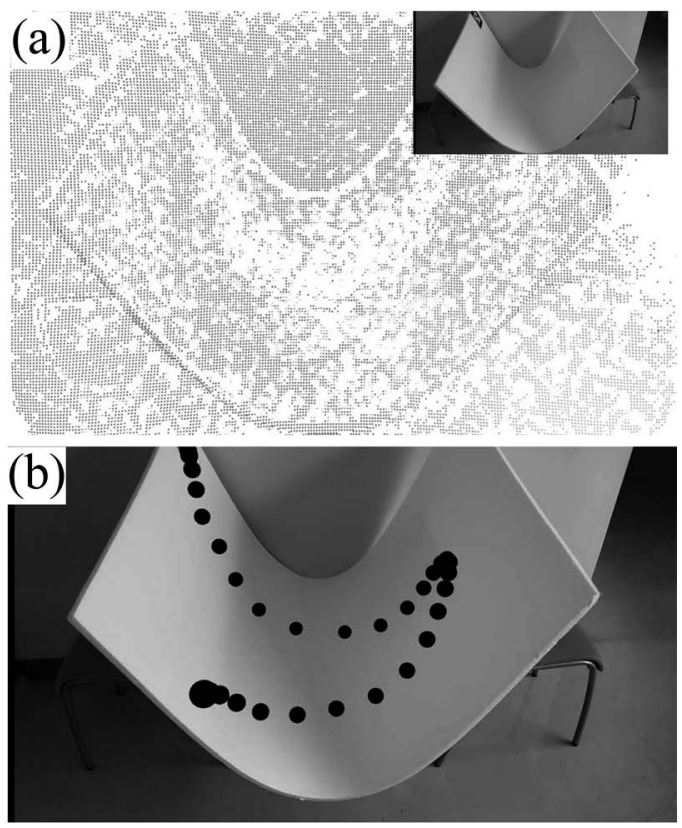

Figure 1. Point Cloud Data of the Potential Shown in Inset (a), Motion of AR Sphere within Potential (b). tional acceleration within UNITY space was $9.8 \times 10^{-2} \mathrm{~m} / \mathrm{s}^{2}$, which is $1 / 100$ of the actual gravitational acceleration. When the AR sphere is set in motion, a point is marked at the position of the AR sphere at 1 -second intervals (Figure 2). Given that the gravitational acceleration within the AR space is negligible, the changes in the motion of the AR sphere can be easily observed in real time. For example, for a $1.8-\mathrm{m}$ free fall, the actual time required is $0.6 \mathrm{~s}$, but it takes $6 \mathrm{~s}$ in AR.

The distance traced by the AR sphere at 1-second intervals is shown in the left side of the screen (Figure 2(a)). The first line of the table indicates the elapsed time, and the second line indicates the moving distance in real space.
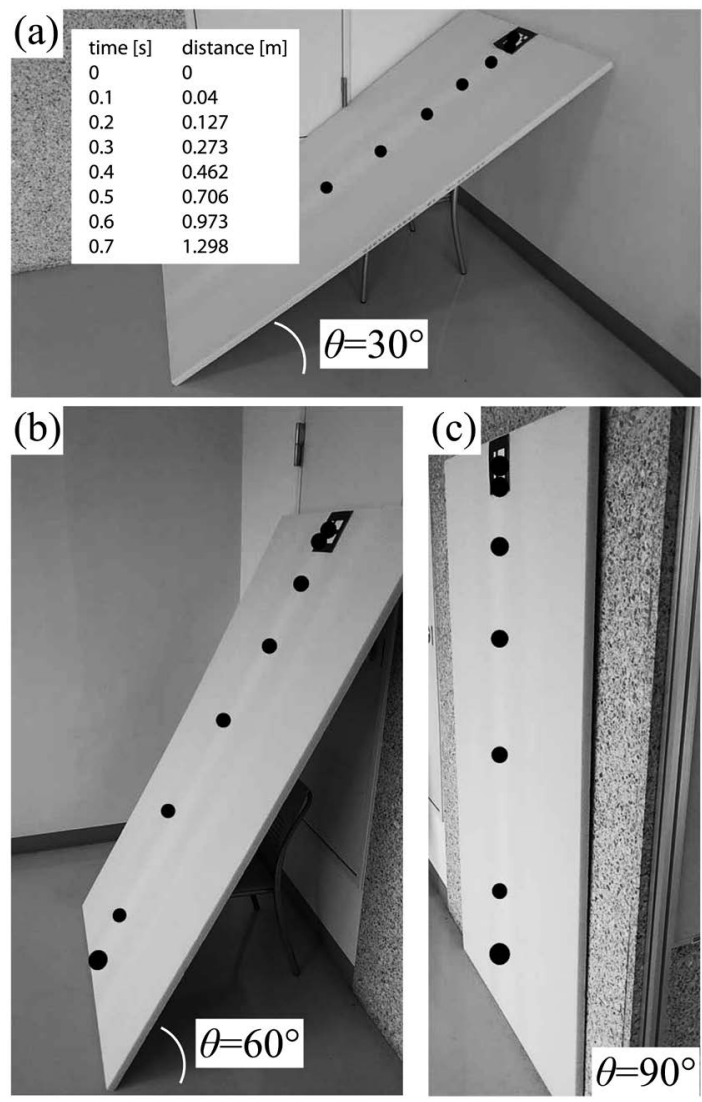

Figure 2. Measurement Results for $\theta=30^{\circ}$ (a), $60^{\circ}$ (b), and $90^{\circ}$ (c). 


\subsection{Potential Slope}

As the potential shape to evaluate the teaching aid, we used a foam-based heat insulating plate of $1820 \times 910 \times 25 \mathrm{~mm}($ length $\times$ width $\times$ thickness $)$, that can be procured from a home repair center. The specific positions of the markers, which are printed on A4 size sheets, are determined along the edge of the slope. The initial position of the AR sphere is superimposed on the markers.

\section{Evaluation of Proposed Teaching Aid}

To evaluate the measurement accuracy of the proposed teaching aid, a 1.8-m long slope with a slope angle of $\theta=30,60$, and $90^{\circ}$ was used (Figure 2). The real space potential was scanned from a distance about $2 \mathrm{~m}$ away from the slope. Figure 3(a) shows the time dependences of the distance, while Figure 3(b) shows the
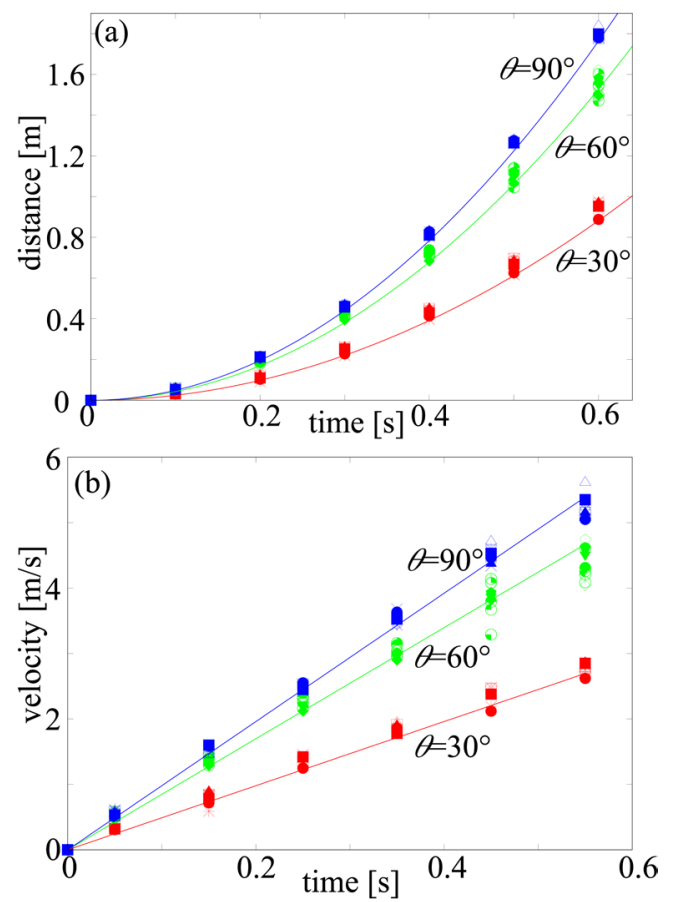

Figure 3. Changes in Moving Distance (a) and Velocity (b) of AR Sphere for $\theta=30,60$ and $90^{\circ}$. The Solid Lines Indicate the Ideal Curves. velocity for each $\theta$ at 10 measurement times. The position of the AR sphere, moving under 1/100 gravity, was tracked at 1-second intervals; however, the frame rate supported by the software during motion was restricted to 12 to $15 \mathrm{fps}$ ( 83 to $67 \mathrm{~ms}$ ). Therefore, the largest time error at each point was $1 \pm 0.083 \mathrm{~s}$. At $\theta=90^{\circ}$, the spatial perception of the potential shape did not affect the measurement. The measurement errors were determined using the distance measurement error due to the depth camera and the time error. The root mean square (RMS) deviation in the velocity was observed to be $6 \%$. As the value of $\theta$ decreased, the error of the spatial perception of the potential shape increased; the RMS deviation in velocity was $10 \%$ at $\theta=60^{\circ}$ and $14 \%$ at $\theta=30^{\circ}$. At $\theta<5^{\circ}$, IMU could not detect the potential as the slope, and the motion of the AR sphere was not accurate at the slope.

\section{Discussion}

We discuss the educational effect of this teaching aid compared with that of the dynamics cart. Conventionally, the following two basic configurations are studied using a dynamics cart in elementary mechanics.

Property I: The velocity of the object on the linear slope is proportionally changed with time.

Property II: The velocity is changed with the slope angle.

According to property I, recording the distance on the tape at the fixed time intervals is known as the appropriate method. This teaching aid is considered to serve the same function as the tape measurement using the dynamics cart. Regarding property II, since the velocity difference between three typical angles $\left(30,60\right.$ and $\left.90^{\circ}\right)$ can be clearly measured within the measurement error, this teaching aid is considered to be useful for learners for studying about the slope dependence of the velocity. Combining the above two concepts, learners try to understand the dynamics in the freely set three-dimensional (3D) shape potential, but the experiment cannot proceed using the dynamics cart, because the measurement is limited to the unidirectional motion. Using this teaching aid, learners can tell the dynamic motion in the $3 \mathrm{D}$ potential as AR, intuitively.

\section{Summary and Future Work}

In this study, we developed a teaching aid for 
dynamics, wherein an AR sphere experienced motion within real potential. The proposed system was implemented using the Tango platform, which is a markerless AR device.

We conjecture that the proposed aid will find applications in middle school science classes, high school physics classes, and college-level dynamics classes. In our future work, we intend to develop software suited for $9^{\text {th }}$ grade middle school students, and conduct classes using about 10 Tango units.

Marker-less AR devices are expected to take on irreplaceable roles in technology enabled learning initiative. The development of Tango was ended in March 2018, and the development of marker-less AR is being continued as ARCore by Google. A similar system is released by Apple as ARKit ${ }^{(11)}$. The necessity of spatial perception technology is increasing for utilization in face authentication, therefore, we expect that the realization of a wide range of education applications based on $\mathrm{AR}$ will be possible.

\section{Acknowledgement}

This work was supported by JSPS KAKENHI Grant Number JP17K04844, and by the Telecommunications Advancement Foundation.

\section{References}

(1) Yuen, S. C.-Y., Yaoyuneyong, G. and Johnson, E.: "Augmented Reality: An Overview and Five Directions for AR in Education", Vol. 4, No 1, pp.119-140 (2011).
(2) Kesim, M. and Ozarslan, Y.: "Augmented Reality in Education: Current Technologies and the Potential for Education", Procedia Social and Behavioral Sciences, Vol. 47, pp. 297-302 (2012).

(3) Lee, K.: "Augmented Reality in Education and Training", TechTrends, Vol. 56, No 2, pp. 13-21 (2012).

(4) Aoki, Y.: "Review of Augmented and Virtual Reality for Middle School Science Education", Proc. of International Conference on Technology and Social Science 2018, http://conf.e-jikei.org/ICTSS2018/proceedings/index.htm (accessed 2018.5.30)

(5) Aoki, Y. and Imachi, S.: "Development of AR Teaching Material by Tablet for Beginning Students of Sound Wave Propagation and Detection", Proc. of International Conference on Technology and Social Science 2018, http:// conf.e-jikei.org/ICTSS2018/proceedings/index.htm (accessed 2018.5.30)

(6) AR text: "Tokyo Shoseki", https://ten.tokyo-shoseki.co.jp/ text/hs/math/book001/level5/index.htm (accessed 2018.5.30)

(7) HoloLens: "Microsoft HoloLens", https://www.microsoft. com/en-us/hololens (accessed 2018.5.30)

(8) Hanna, M. G., Ahmed, I., Nine, J. et al.: “Augmented Reality Technology Using Microsoft HoloLens in Anatomic Pathology", Archives of Pathology \& Laboratory Medicine, Vol. 142, pp. 638-644 (2018).

(9) ARCore: "Google Developers", https://developers.google. com/ar/ (accessed 2018.5.30)

(10) Zenfone AR: “ASUS Zenfone AR", https://www.asus. com/Phone/ZenFone-AR-ZS571KL/ (accessed 2018.05.30)

(11) ARKit: "Apple Developers", https://developer.apple.com/ jp/arkit/ (accessed 2018.5.30) (in Japanese) 